\title{
CSR- und Nachhaltigkeitsmanagement: Beiträge der Forschung und Praxis
}

\section{Vorwort der Herausgeber}

\author{
Alexander Moutchnik ${ }^{1}$ - René Schmidpeter ${ }^{2}$
}

(C) Springer-Verlag Berlin Heidelberg 2016

Vom 1. bis zum 3. August 2016 fand in Köln die dritte „International Conference on CSR, Sustainability, Ethics and Governance“ mit dem Fokus auf „Sustainability as New Business Paradigm“statt. Dieses Forum für umwelt-, nachhaltigkeits- und CSR-fokussierte Wissenschaftler und Praktiker aus mehr als vierzig Ländern wurde von der Cologne Business School und dabei insbesondere vom Dr. Jürgen Meyer Stiftungslehrstuhl für Internationale Wirtschaftsethik und CSR sowie vom Global Corporate Governance Institute (www.gcg-csr.org) ausgerichtet. Nach den erfolgreichen Konferenzen in London (2014) und Nanjing (2015) bekam diese nunmehr in einem jährlichen Turnus stattfindende Tagung einen großen Zuspruch von der internationalen CSR-Forschung und -Praxis. Das Ziel, die Wissenschaftler aus allen Bereichen mit Führungspersonen aus Wirtschaft, Kultur, Religion, Politik und der akademischen Welt zusammenzuführen, um eine globale Allianz zu bilden, welche der Integration von Wertfragen in Management-Praxis, Bildung und Entwicklung dient, wurde auch im Jahr 2016 mit großem Erfolg erreicht.

Die vierte „International Conference on CSR, Sustainability, Ethics and Governance" wird vom 26. bis zum 28. Juli 2017 in Perth (Westaustralien) stattfinden und wird dem Thema „Responsible Business for Uncertain Times and a Sustainable Future“ gewidmet. Die Abstracts können noch bis zum 28. Februar 2017 auf der Internetseite der Tagung (www.promaco.com.au/events/csr2017/index.php) hochgeladen werden.

Alexander Moutchnik

alexander.moutchnik@hs-rm.de

1 Hochschule RheinMain, Wiesbaden, Deutschland

2 Cologne Business School, Köln, Deutschland
Die Beiträge der Teilnehmer der Kölner Konferenz des Jahres 2016 werden in Sammelbänden der SpringerBuchreihe „CSR, Sustainability, Ethics \& Governance“, im neu erscheinenden ,Journal of Corporate Governance“ (Emerald) und „International Journal of Corporate Social Responsibility“ (Springer) sowie in dieser Ausgabe der Zeitschrift „UmweltWirtschaftsForum“ (Springer) veröffentlicht.

Hans Verboven und Lise Vanherck von der Universiteit Antwerpen beschäftigen sich in ihrem Beitrag zum Thema „The sustainability paradox of the sharing economy“ nicht etwa mit den positiven Effekten sogenannter nachhaltiger Geschäftsmodelle wie z. B. Sharing Economy, sondern mit solchen Geschäftsmodellen, die auch unbeabsichtigte, negative Externalitäten aufweisen. Sie seien weniger sichtbar und werden oft ignoriert. In ihrem Beitrag definieren Verboven und Vanherck die Folgen solcher negativen Nebenwirkungen als Nachhaltigkeitsparadox und beschreiben die negativen Externalitäten der Sharing Economy durch den Vergleich mit den wichtigsten Eigenschaften eines nachhaltigen Wirtschaftsmodells. Mit der aufgestellten Checkliste zur schnellen Identifizierung von Nachhaltigkeitsmerkmalen in neuen Geschäftsmodellen können zum einen legislative Initiativen von oben nach unten umgesetzt und zum anderen die Geschäftsmodelle mit der weitgehenden Vermeidung von Externalitäten gestaltet werden.

Anne Michaels und Michael Grüning von der Technischen Universität Ilmenau untersuchen in ihrem Beitrag empirisch den Einfluss von Corporate Identity auf Publizität im Bereich unternehmerischer Verantwortung bzw. Corporate Social Responsibility. Ihre Untersuchung basiert auf einer Stichprobe von 498 Unternehmen in Deutschland, die englischsprachige CSR-Informationen veröffentlichen und einen Fragebogen beantworten, der den Grad von CSRorientierter Corporate Identity misst. Die CSR-Publizität 
werteten Michaels und Grüning mit Hilfe einer automatisierten Inhaltsanalyse unter Anwendung künstlicher Intelligenz aus. Die Ergebnisse zeigen, dass bestimmte Corporate Identity-Dimensionen, welche mit der Wertschöpfungskette enger verbunden sind, CSR-Publizität begünstigen, während nach innen gerichtete Corporate Identity-Dimensionen eindämmend wirken. Die Verfasser demonstrieren, dass die Ausprägung CSR-orientierter Corporate Identity eng mit dem Entwicklungsgrad von CSR im Unternehmen und dem empfundenen Rechtfertigungsdruck verknüpft ist. Die Studie von Michaels und Grüning unterstützt die Sichtweise, dass eine CSR-orientierte Corporate Identity, welche in der Literatur oft als Luxus erfolgreicher Unternehmen angesehen wird, eine Verbesserung der CSR-Publizität ermöglicht, die wiederum finanzielle und immaterielle Vorteile nach sich ziehen kann.

Carsten Deckert von der Cologne Business School geht in seinem Beitrag zum Thema „Ecological Sustainability of Material Resources - Why Material Efficiency just Isn't Enough" auf die Unterschiede zwischen der Wirtschaft mit relativ knappen Ressourcen und der Wirtschaft mit absolut knappen Ressourcen mit Schwerpunkt auf materielle Ressourcen ein. Deckert vertritt die Meinung, dass die Nachhaltigkeitsforschung ihren Fokus von einer bloßen Erhöhung der Materialeffizienz zu den Problemen der Ressourcenverantwortung und der Erhaltung der natürlichen Ressourcen unter systemdynamischer Perspektive ändern muss.

Bing Zhu von der Katholischen Universitat Eichstatt-Ingolstadt analysiert in ihrem Beitrag zum Thema „Consumer's Motivation, Opportunities and Ability for Sustainable Consumption“ auf Basis des Motivation-Opportunity-Ability-Modells jene Faktoren, welche den Konsum organischer Lebensmittel in Südchina am meisten beeinflussen.

Stephanie Bothe, Dorothea Schaffner, Uta Jüttner und Lutz Schlange von der Hochschule Luzern untersuchen in ihrer Arbeit die Qualität nachhaltiger Dienstleistungen aus Kundensicht sowie ihren Einfluss auf die Kundenzufriedenheit und die Absicht der Kunden, sich in die Dienstleistungserstellung zu integrieren. Basierend auf bestehenden Theorien stellen die Verfasser eine dreidimensionale Struktur für die Qualität nachhaltiger Dienstleistungen dar: Zusätzlich zu den bekannten faktischen und emotionalen Qualitätsdimensionen führen Bothe, Schaffner, Jüttner und Schlange eine normative Dimension ein. Die empirische Überprüfung erfolgt für eine Carsharing- und eine Recycling-Dienstleistung. Die qualitative Untersuchung bestätigt die dreidimensionale Struktur der Qualität nachhaltiger Dienstleistungen. Die quantitative Untersuchung zeigt ihren positiven Einfluss auf Kundenzufriedenheit und die Mitgestaltungsabsicht der Kunden. Der Beitrag zeigt deutlich, dass Konsumenten Dienstleistungen nutzen und kaufen wollen, die ihre moralischen Werte bezüglich Nachhaltigkeit unterstützen und ein positives emotionales Erlebnis hervorrufen, jedoch gleichzeitig keinerlei funktionale Nachteile im Vergleich zu nicht nachhaltigen Dienstleistungsalternativen aufweisen.

Wolfgang Keck von der Kommunikationsagentur ,keck kommuniziert!“ geht in seinem Beitrag auf die folgende Frage ein: „Do we really think small first? How to go further with CSR on micro enterprises“. Der Beitrag liefert ein umfassenderes Bild von CSR in Kleinstunternehmen und präsentiert Ideen zur Ermutigung von Kleinstunternehmen, sich intensiv mit den Fragen des nachhaltigen Managements auseinanderzusetzen, denn die kleinsten Unternehmen seien mit Abstand die größte Unternehmensgruppe in der Europäischen Union.

Laura Bauer und Holger Schunk von der Hochschule RheinMain in Wiesbaden untersuchen „Markenführung und Nachhaltigkeit in der Textilindustrie" und gehen dabei insbesondere auf die Kaufentscheidung bei Textilien ein. Darüber hinaus diskutieren Bauer und Schunk die Verankerung von Nachhaltigkeit in der Markenidentität und die daraus resultierende Markenwahrnehmung.

Laura Marie Schons und Sabrina Scheidler von der Universität Mannheim stellten in ihrem Ansatz die ,Forschungskooperationen zwischen Wissenschaft und Praxis zum Thema ,Corporate Social Responsibility ‘ am Beispiel der IKEA Deutschland“ dar. Dieser Beitrag beschäftigt sich am Beispiel anhand der mit dem Wissenschaftspreis 2016 ausgezeichneten Forschungskooperation zwischen IKEA und einer Forschergruppe damit, wie solche Kooperationen ausgestaltet sein könnten, um für beide Parteien gewinnbringende Erkenntnisse zu generieren. Insbesondere wird dabei die wichtige Rolle von Feldexperimenten beleuchtet. Der Artikel schließt mit zehn Erfolgsfaktoren für eine erfolgreiche Zusammenarbeit zwischen Wissenschaft und Unternehmenspraxis.

Suppasit Sornsri von der Assumption University - Bangna Campus in Thailand erörtert in seinem Beitrag die Umsetzungsmöglichkeiten des philosophischen Ansatzes der Suffizienz-Ökonomie bei der Organisation des Einkaufs in Unternehmen. Dieser Ansatz spiegelt die Grundsätze der thailändischen Kultur wieder und bezieht sich auf die MäBigung, die Vernunft und die Selbstimmunität.

Enes Ünal, Vikash Kumar Sinha und Davide Chiaroni (Politecnico di Milano) gehen in ihrem Beitrag „Operationalization strategy of sustainability priorities in the phase of transition to circular economy: The case of Cradle to $\mathrm{Cr}$ adle (C2C)" auf das Konzept von Cradle to Cradle (C2C) im Rahmen des Übergangs zur Kreislaufwirtschaft ein. Die Verfasser haben insgesamt 115 Produkte auf (1) Materialgesundheit, (2) Materialverwendung, (3) Erneuerbare Energien, (4) Water Stewardship und (5) Soziale Fairness systematisch analysiert, um zu verstehen, ob eine Priorisierung einer bestimmten Praxis unter den Branchen vorliegt. 
Die Ergebnisse zeigen, dass Unternehmen gleichzeitig allen umweltpolitischen Prioritäten nachgehen.

Alexander Moutchnik von der Hochschule RheinMain in Wiesbaden analysiert in seinem Beitrag die Gründe für Kirchen und andere religiöse Organisationen und Institutionen, international geltende Normen für Qualitäts- und Umweltmanagementsysteme (ISO 9001, ISO 14001 und ISO 26000) zu implementieren.

Integration Viola Muster, Ulf Schrader, Susanne Blazejewski, Martina Schäfer, Anke Buhl, Christoph Harrach, Marc Schmidt-Keilich und Elisabeth Süßbauer von der Technischen Universität Berlin (Institut für Berufliche Bildung und Arbeitslehre, Zentrum Technik und Gesellschaft) und von der Alanus Hochschule für Kunst und Gesellschaft (Lehrstuhl für nachhaltige Organisations- und Arbeitsplatzgestaltung) präsentieren in ihrem Beitrag mit der Integration von (nachhaltigkeitsorientierten) Mitarbeitern in ihrer Konsumentenrolle einen neuen Ansatz. Dieser steht im Mittelpunkt des vom Bundesministerium für Bildung und Forschung finanzierten Verbundprojekts IMKoN (Integration von Mitarbeitern als Konsumenten in Nachhal- tigkeitsinnovationsprozesse). Als erstes Teilergebnis stellt das Verfasser-Team die Chancen und Risiken des IMKoNAnsatzes am Beispiel einer Bestandsaufnahme bei acht Unternehmen unterschiedlicher Größe aus verschiedenen Branchen dar.

Diese Ausgabe der Zeitschrift „UmweltWirtschaftsForum" entstand vor allem durch die engagierte Mitarbeit aller Autoren, denen für ihren Einsatz sehr herzlich gedankt wird. Ein Dank gilt auch dem Schriftleiter der Zeitschrift, Professor Dr. Dietfried Günter Liesegang, für die Aufnahme des Themas als Schwerpunkt in die UmweltWirtschaftsForum-Reihe.

Danken möchten wir auch Thomas Tschech, dem Production Editor des Springer-Verlags für die freundliche und fachkundige Unterstützung bei der Herausgabe dieser Zeitschrift.

Allen Leserinnen und Lesern wünschen wir eine anregende und spannende Lektüre dieser Ausgabe des UmweltWirtschaftsForums.

Alexander Moutchnik, René Schmidpeter

Wiesbaden-Köln, Dezember 2016 